\title{
A Fuzzy Optimisation Model with Applications to Air Pollution Exposure Mitigation
}

\author{
$\underline{\text { L. Schultz }}^{\mathrm{a}}$, J. Boland ${ }^{\mathrm{a}}$, P. Shah ${ }^{\mathrm{b}}$ and B. Chiera ${ }^{\mathrm{a}}$ \\ ${ }^{a}$ Centre for Industrial and Applied Mathematics, School of Information Technology and Mathematical \\ Sciences, University of South Australia \\ ${ }^{b}$ Air and Noise Group, Science and Assessment Division, South Australian Environment Protection Authority \\ Email: lisa.schultz@unisa.edu.au
}

\begin{abstract}
Exposure to air pollution has been extensively associated with adverse health effects, including cardiovascular illness and premature mortality. To reduce the risk of exposure, Australian state governments develop candidate mitigation strategies outlining specific actions and interventions to reduce emissions of, and exposure to, air pollutants. However, a common challenge in air quality management lies in selecting the optimal control strategies from a potentially broad range of candidate strategies to mitigate exposure whilst adhering to budgetary requirements and national air quality standards. The complexity of this challenge is further compounded by the many different, incomparable types of uncertainty inherent to air quality analyses.
\end{abstract}

To this end, optimisation models have been a commonly used tool in air quality management. However the majority of optimisation models presently available in the literature typically focus on minimising implementation costs alone, with limited consideration of uncertainty in model parameters.

In this paper, a theoretical optimisation model is formulated and solved to accommodate for uncertainty to some extent in model parameters and the optimal solution. The developed model is applied in an air pollution context to identify cost-effective strategies to potentially reduce pollution exposure, with the objective of maximising the estimated societal health benefits, rather than implementation costs alone, under uncertainty. Within the model, the inherent spatiotemporal uncertainty of emission analyses can be represented, to an extent, through the combination of traditional linear programming techniques with the principles of fuzzy logic. This is an attractive modelling approach as fuzzy logic allows for imprecise model parameters.

The developed model is solved using a fuzzy linear complementary problem approach with Lemke's pivoting algorithm. The numeric model output is complemented with maps of the model results, including health impact maps showing the expected number of adverse health episodes potentially avoided through the implementation of the optimal solution. These maps allow for simple, quick interpretation of the model results and easy identification of areas with the greatest, and least, potential for health impact.

The use and format of model results are demonstrated through an application to Adelaide, South Australia, under sample emission scenarios. The performance of the developed fuzzy model is evaluated through a comparison against the corresponding 'crisp' model wherein all model parameters are assumed to be precisely defined. The results indicate that the fuzzy model is able to identify a cost-effective control strategy, or combination of strategies, to potentially reduce pollution exposure within the limited context of the sample application. Results are consistent between the developed fuzzy model and the corresponding crisp model however the fuzzy model has the additional advantageous feature of representing parameter uncertainty to some extent through the provision of upper and lower limits on the model results.

There are complex processes beyond the scope of the developed theoretical model which impact on emission analyses. However the developed optimisation model is a step towards an accountable framework for air quality managers to aid the selection of cost-effective mitigation strategies that maximise the health benefits to society while accommodating, to an extent, for the associated uncertainty.

Keywords: Air pollution, optimisation, fuzzy logic, exposure mitigation 


\section{INTRODUCTION}

Exposure to traffic-related air pollution has been extensively associated with adverse health effects, including diabetes incidence, impaired lung function and growth, cognitive impairment and premature mortality (Gauderman et al., 2004; Power et al., 2011; Raaschou-Nielsen et al., 2013). According to the World Health Organisation, ambient outdoor air pollution was responsible for 3 million premature deaths in 2012 (World Health Organisation, 2017). To mitigate the risk of exposure to harmful air pollution and protect public health, air quality managers develop exposure mitigation strategies outlining specific actions and interventions to reduce air pollution concentrations. A prominent challenge in air quality management therefore lies in the selection of the most economic and effective control strategies from a potentially broad range of candidate strategies. This challenge is further complicated by local emission targets and budgetary constraints, which impact the control strategy selection process, as well as the many different types of incomparable uncertainty inherent to air quality analyses (Oxley and ApSimon, 2011). For example, sources of uncertainty in air quality analyses include, but are not limited to, the estimated efficacy and cost of a candidate control strategy as well as the associated expected health impact, which may not be explicitly quantifiable or fully realised prior to implementation. Moreover, estimated air pollution concentrations are typically based on mathematical models which are inherently subject to uncertainty (Schultz, 2016). A detailed conceptualisation of the many different sources of uncertainty which arise in air quality analyses can be found in Oxley and ApSimon (2011).

To aid air quality managers in selecting economic and effective mitigation strategies, a number of optimisation models are available in the literature (Liu et al., 2003; Lv et al., 2011; Qin et al., 2010; Sonawane et al., 2012). These models have differing levels of complexity however are typically formulated to minimise the costs associated with implementation of each candidate control strategy. At the time of this research, there are limited models available which explicitly account for the expected health benefits associated with each control strategy whilst handling and representing the uncertainty in model parameters. This is an important consideration in air quality cost-benefit analyses as the control strategies with the lowest implementation costs may not be as effective at reducing long-term incidence rates of adverse health outcomes as more costly control strategies, leading to an increase in long-term health expenses (Schultz, 2016). Moreover, accommodation of uncertainty in the model is imperative to ensure air quality management decisions are well-informed and accountable.

In this paper, a new theoretical optimisation model is formulated and solved in the presence of uncertainty with applications to selecting optimal control strategies to potentially mitigate air pollution exposure. The objective of the developed model balances the trade-off between implementation costs and the expected health benefits to society. This objective has been established on the basis of the optimisation model proposed in Sonawane et al. (2012), in which health benefits to society are a key model parameter in identifying optimal control strategies for traffic-related air pollution. A distinguishing feature of the developed fuzzy health benefits model lies in the combination of traditional optimisation techniques with fuzzy logic, used to represent the uncertainty associated with model parameters to some extent. Fuzzy optimisation is an attractive modelling choice as it allows model parameters to be imprecise, such as monetary values which are subject to frequent temporal variation. It should be noted that there are a number of complex processes impacting air pollution emissions and exposure that are beyond the scope of the present research. The developed fuzzy model is therefore a step towards an accountable framework for the management of air quality which accommodates for uncertainty.

The layout of this paper is as follows. First, preliminary information on the concept of a fuzzy number is provided in Section 2, with these concepts then applied in Section 3 to formulate the fuzzy health benefits optimisation model. The method used to solve the developed fuzzy optimisation model is described in Section 4, followed by a demonstration of the model use and format of the results through an application to Adelaide, South Australia, with two theoretical control strategies in Section 5. Conclusions are drawn on the performance of the developed fuzzy optimisation model in Section 6. This research was conducted as part of the doctoral dissertation of the lead author, and the interested reader can find further details on this work in Schultz (2016).

\section{PRELIMINARIES}

The fuzzy optimisation model developed here combines traditional optimisation techniques with the principles of fuzzy logic to represent uncertainty in model parameters. A number is considered to be 'fuzzy' if it is imprecisely defined and is supported over a finite interval of numbers (Lai and Hwang, 1992). For example, health effect estimates associated with air pollution exposure are commonly reported as a mean effect estimate $\bar{x}$ with an accompanying $95 \%$ confidence interval. This mean effect estimate $\bar{x}$ can be considered as a fuzzy number supported over the finite interval spanned by the $95 \%$ confidence interval.

A general fuzzy number is denoted $\tilde{A}=\left(a_{1}, a_{2}, a_{3}\right)$, with $a_{2}$ representing the centre of the fuzzy set and $a_{1}$ and $a_{2}$ as the lower and upper bounds of the fuzzy set, respectively. Here, the $\sim$ accent indicates that $A$ is a 
fuzzy number. All fuzzy numbers have an accompanying membership function $\mu(x)$ which maps $x \in \mathbb{R}$ to the interval [0,1], with values of 1 indicating that $x$ is completely within the fuzzy set for $\tilde{A}$ and values of 0 indicating that $x$ is not an element of $\tilde{A}$. These membership functions can take a number of forms, depending on the context of the fuzzy number being represented. Following the established convention in the literature for numbers that are reported with an accompanying 95\% confidence interval (Mesa-Frias et al., 2014), the triangular membership function has been used here, given by

$$
\mu(x)=\left\{\begin{array}{lc}
\left|\frac{x-a_{1}}{a_{2}-a_{1}}\right| \quad & \text { if } a_{1} \leq x \leq a_{2} \\
\left|\frac{a_{3}-x}{a_{3}-a_{2}}\right| & \text { if } a_{2} \leq x \leq a_{3} \\
0 & \text { if } x \geq a_{3} \text { or } x \leq a_{1} .
\end{array}\right.
$$

Here, $a_{1}$ and $a_{3}$ can be considered to be the lower and upper bounds of the $95 \%$ confidence interval, respectively, with $a_{2}$ as the mean effect estimate. As an example, Hansen et al (2012) report an increase of $4.48 \%$ in the odds of cardiovascular hospital admissions during cool seasons for a $10 \mu \mathrm{g} / \mathrm{m}^{3}$ increase in ambient particulate matter, with a $95 \%$ confidence interval of $(0.74 \%, 8.36 \%)$. A fuzzy representation of this effect estimate is $\tilde{A}=(0.74,4.48,8.36)$, with $\mu(4.48)=1$ and $\mu(0.74)=\mu(8.36)=0$, as shown in Figure 1 .

In the developed fuzzy optimisation model, fuzzy numbers are used to represent uncertain model parameters. A critical component in this optimisation model lies in the comparison of two fuzzy numbers to ensure model constraints are satisfied. In practice, the comparison of two fuzzy numbers is achieved through the use of a ranking function $\mathcal{R}$ mapping a fuzzy number to the real line $\mathbb{R}$ (Mottaghi et al., 2015). A number of ranking functions are available in the literature, each of which satisfy the following properties (Mottaghi et al, 2015): for two fuzzy numbers $\tilde{A}$ and $\tilde{B}, \tilde{A} \geq \tilde{B}$ if and only if $\mathcal{R}(\tilde{A}) \geq \mathcal{R}(\tilde{B})$; and $\tilde{A}=\tilde{B}$ if and only if $\mathcal{R}(\tilde{A})=\mathcal{R}(\tilde{B})$. Here, the ranking function of Facchinetti et al (1998) is used, where

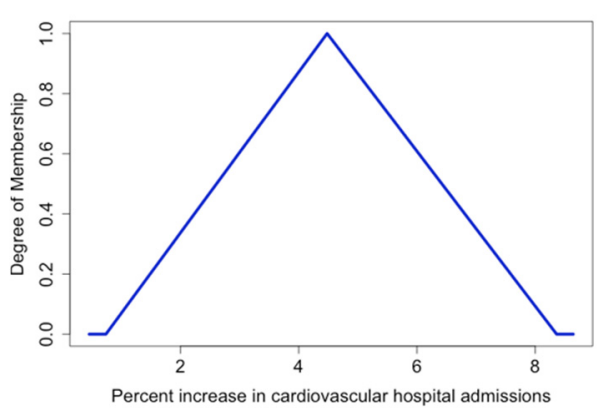

Figure 1. Example fuzzy representation of a mean effect estimate and the accompanying $95 \%$ confidence interval.

$$
\mathcal{R}(\tilde{A})=\frac{a_{1}+2 a_{2}+a_{3}}{4} .
$$

This is a flexible ranking function that is particularly sensitive to the spread of a fuzzy number and allows for the comparison of a fuzzy number with a crisp, precisely defined number. The interested reader can find further technical details on the theory and use of fuzzy numbers in Lai and Hwang (1992).

\section{FORMULATION OF THE FUZZY HEALTH BENEFITS OPTIMISATION MODEL}

The developed fuzzy health benefits optimisation model is formulated to select the optimal exposure mitigation strategy, or combination of strategies, to reduce the potential for population exposure to traffic-related air pollution in the presence of uncertainty. The objective of the developed model therefore balances the trade-off between the costs associated with implementing the candidate control strategies and the resulting economic value of the health benefits to society, with the uncertainty inherent to emission analyses and health impact assessments captured using the principles of fuzzy logic (\$2). The use of fuzzy logic allows parameter estimates to be imprecise or not fully realised, such as monetary values which are subject to frequent temporal variation.

In the objective function of the optimisation model, the economic health benefits to society are estimated by combining the expected number of adverse health incidents avoided as a result of each candidate control strategy with the economic value of an individual episode of each health endpoint. First, the expected number of avoided adverse health incidents is estimated using a concentration-response function, $\Delta y\left(h ; x_{i}\right)$,

$$
\Delta y\left(h ; x_{i}\right)=\tilde{x}_{i}\left(1-e^{-\widetilde{\beta}_{h} \Delta P M_{i}}\right) I_{h} P .
$$

Here, $\tilde{x}_{i}$ is the proportion of implementation of control strategy $i$, to be determined by the developed optimisation model, $\triangle P M_{i}$ is the expected change in air pollution associated with the $i^{\text {th }}$ candidate control strategy, $I_{h}$ is the baseline incidence rate of adverse health endpoint $h$ and $P$ is the total population in the area of analysis. Following convention in the literature, particulate matter (PM) is used as the target pollutant in the developed optimisation model (Abt Associates Inc., 2012; Hansen et al., 2012; Simpson et al., 2005). 
Additional pollutants can be added to the model subject to the needs and interests of the users. The interested reader can find further technical information on the derivation of model parameters in Schultz (2016).

In (3), the $\tilde{\beta}_{h}$ coefficient is the key parameter governing the accuracy and reliability of the resulting estimated number of avoided health incidents. This coefficient represents the relationship between a one unit change in ambient air pollution levels and the incident rate of health effect $h$. There are a number of studies available in the literature for deriving $\tilde{\beta}_{h}$ coefficients, however the corresponding values of $\tilde{\beta}_{h}$ differ substantially depending on the location and assumptions of the parent study (Schultz, 2016). As such, the $\tilde{\beta}_{h}$ values used here were sourced from a recent domestic study of the risks associated with air pollution exposure (Golder Associates, 2013). These $\tilde{\beta}_{h}$ values were compiled based on meta-analyses of high calibre domestic and international research and are therefore considered best practice for use in the developed optimisation model in estimating the number of health incidents avoided through the candidate control strategies. To capture the uncertainty in health estimate effects, the $\tilde{\beta}_{h}$ values were represented as fuzzy numbers, using the $95 \%$ confidence intervals accompanying the mean effect estimates as the bounds of the fuzzy representation (\$2).

The associated cost benefits to society are then approximated by combining (3) with $\tilde{V}_{h}$, the monetary value of an individual episode of adverse health endpoint $h$, and summing over all candidate control strategies $i=1, \ldots, n$ and health endpoints of interest, $h=1, \ldots, H$, as follows

$$
\text { Cost benefits }=\sum_{i=1}^{n} \sum_{h=1}^{H} \tilde{x}_{i}\left(1-e^{-\widetilde{\beta}_{h} \Delta P M_{i}}\right) I_{h} P \tilde{V}_{h} .
$$

Assigning a monetary value $\tilde{V}_{h}$ to an individual episode of an adverse health endpoint $h$ is a complex, uncertain process as there are a number of costs associated with impaired health. These costs include direct medical expenses as well as the less tangible costs associated with personal suffering (Department of Environment and Conservation, 2005). The values of $\widetilde{V}_{h}$ used in the development of the fuzzy health benefits optimisation model were sourced from a number of recent major Australian studies (Australian Safety and Compensation Council, 2008; Boulter and Kulkarni, 2013; Department of Environment and Conservation, 2005). To accommodate for the inherent uncertainty, monetary valuations of health effects are typically reported as $95 \%$ confidence intervals which are used here to represent the monetary estimates as fuzzy numbers $(\S 2)$.

Using the expected health benefits to society calculated in (4), the objective function of the developed fuzzy health benefits optimisation model has been formulated as

$$
\underset{\tilde{x}_{i}}{\widetilde{a}}\left\{\sum_{i=1}^{n} \sum_{h=1}^{H} \tilde{x}_{i}\left(1-e^{-\widetilde{\beta}_{h} \Delta P M_{i}}\right) I_{h} P \tilde{V}_{h}-\tilde{x}_{i} C_{i}\right\} .
$$

Here, $\tilde{x}_{i}$ are the decision variables over which the maximisation is performed, representing the proportion of implementation of the $i^{\text {th }}$ control strategy for $i=1, \ldots, n$, with $C_{i}$ representing the associated implementation costs. Thus the objective function maximises the overall benefits to society by maximising the difference between the estimated health benefits from (4) and the implementation costs of each candidate control strategy. In (5), the max operator indicates that this maximisation involves fuzzy parameters, with the uncertainty associated with the model parameters represented throughout the optimisation procedure.

\subsection{Model Constraints}

The developed fuzzy health benefits model has been formulated with three example model constraints relating to the total budget, emissions targets and standard non-negativity constraint. These are summarised as below.

1. A fuzzy budgetary constraint ensures that the cost $C_{i}$ of implementing each candidate control strategy $i$ does not exceed the available budget $\widetilde{B}$. This constraint is formalised as fuzzy as the available budget may not be finalised prior to the selection of candidate control strategies,

$$
\sum_{i=1}^{n} C_{i} \tilde{x}_{i} \leq \tilde{B} \text {. }
$$

2. An emissions target constraint to ensure that the reduction in ambient air pollution $\Delta P M_{i}$ achieved as a result of the $i^{\text {th }}$ candidate control strategy is below a given emissions reduction target $E$,

$$
\sum_{i=1}^{n} \Delta P M_{i} \leq E
$$


3. Standard non-negativity constraints to ensure that the optimal implementation proportions $\tilde{x}_{i}$ identified by the fuzzy health benefits optimisation model are valid and sensible. That is, $0 \leq \tilde{x}_{i} \leq 1$ for all $i=1, \ldots, n$.

In practice, the developed health benefits model is flexible in terms of model constraints however, with users able to add or remove constraints as needed (Schultz, 2016).

\section{METHOD OF SOLUTION FOR THE FUZZY HEALTH BENEFITS OPTIMISATION MODEL}

The landscape of solution algorithms for optimisation models which include fuzzy parameters is narrow and highly sensitive to the role of the fuzzy parameters in the model (Lai and Hwang, 1992). In the developed fuzzy health benefits optimisation model, both the objective function and model constraints have fuzzy parameters. This type of fuzzy model, where both the objective function and decision space are subject to uncertainty, can be readily solved using the method proposed in Mottaghi et al. (2015). This method first reformulates the fuzzy health benefits optimisation model as a fuzzy linear complementary problem (Mottaghi et al., 2015), which is then solved using Lemke's complementary pivoting algorithm (Lemke, 1968). The interested reader can find complete technical details on this method of solution in Mottaghi et al. (2015) and Schultz (2016).

\section{SAMPLE DEMONSTRATION OF MODEL USE}

In this section, the use and output of the developed fuzzy optimisation model is demonstrated through a theoretical analysis of Adelaide, South Australia (Figure 2). The results of the fuzzy model are compared and contrasted with the crisp alternative model wherein all model parameters are assumed to be precisely defined integer values. In the crisp model, only the central estimates for the fuzzy model parameters are used, corresponding to the mean effect estimates with the uncertainty of the mean effect estimates not featuring in the crisp model.

Figure 2 shows the $50 \times 50 \mathrm{~km}^{2}$ case study area which has been divided into $1 \mathrm{~km}^{2}$ grid cells, with the developed fuzzy health benefits optimisation model, and the corresponding crisp model, applied separately in each cell to determine the optimal implementation proportions for two candidate control strategies. These strategies have been selected for illustrative purposes, with Control Strategy 2 achieving a greater reduction in emissions $(\triangle P M)$ at a greater cost than Control Strategy 1 . Here, the values of $\triangle P M$ were estimated by first estimating the reduced vehicular emissions of traffic-related air pollutants in the case study area under each control strategy, with The Air Pollution Model

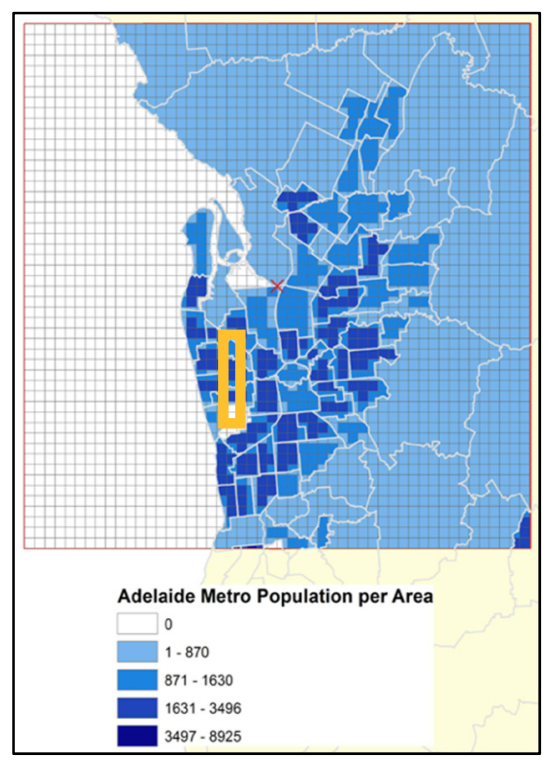

Figure 2. Distribution of the population in the case study area used to demonstrate the developed fuzzy optimisation model. (TAPM) then used to estimate the corresponding ambient air pollution concentrations at baseline as well as under each control strategy (Schultz, 2016). Further technical details are available in Schultz (2016).

A selection of notable results from the crisp and fuzzy optimisation models are shown in Table 1 and Table 2, respectively. These results correspond to the grid cells in rows $31-34$ and 38 of column 21 of the case study area (orange rectangle, Figure 2). For each grid cell, Table 1 and Table 2 provide the optimal implementation proportions of Control Strategies 1 and 2 and the value of the objective function for the crisp and fuzzy optimisation models, respectively, representing the expected societal health benefits. For example, from the crisp version of the health benefits model (Table 1) it can be seen that in the area corresponding to column 21 and row 31 , the optimal solution is to implement $26 \%$ of Control Strategy 2 only. The corresponding health benefits to society is expected to be $\$ 1,341$ in that area. Similar results were obtained from the fuzzy health benefits model (Table 2), however with the optimal solution and objective function represented as fuzzy numbers ${ }^{1}$. The central estimates of the fuzzy optimal solutions $\tilde{x}_{i}^{*}$ for $i=1,2$, and the corresponding value of the objective function $\tilde{z}^{*}$ exactly match the optimal solutions of the crisp model results. However as the fuzzy model accounts for uncertainty, these optimal solutions are reported as uncertain, fuzzy numbers.

${ }^{1}$ Note that in Table 2, the fuzzy numbers $\tilde{x}_{i}^{*}$, for $i=1,2$, represent proportions. As such, the lower limits that are less than zero should be considered as zero and the upper limits greater than one should be treated as one to ensure the results are meaningful. 
Table 1. Select case study results from the crisp optimisation model, where $z^{*}$ is the value of the objective function and $x_{i}^{*}$ is the optimal implementation proportion of the $i^{\text {th }}$ Control Strategy, for $i=1,2$.

\begin{tabular}{c|c|c|c|c|c}
\hline Col. & Row & Population & $\mathbf{z}^{*}$ & $\boldsymbol{x}_{\mathbf{1}}^{*}$ & $\boldsymbol{x}_{\mathbf{2}}^{*}$ \\
\hline 21 & 31 & 1752 & 1341 & 0.000 & 0.26 \\
21 & 32 & 1861 & 1193 & 0.000 & 0.24 \\
21 & 33 & 2171 & 1052 & 0.000 & 0.21 \\
21 & 34 & 2173 & 920 & 0.000 & 0.21 \\
21 & 38 & 0 & 0 & 0.000 & 0 \\
\hline
\end{tabular}

Table 2. A selection of results from the case study for the fuzzy health benefits optimisation model. Here, $\tilde{z}^{*}=\left(z_{1}^{*}, z_{2}^{*}, z_{3}^{*}\right)$ is the value of the fuzzy objective function and the optimal implementation proportions of Control Strategies 1 and 2 are given by $\tilde{x}_{i}{ }^{*}=\left(x_{i, 1}^{*}, x_{i, 2}^{*}, x_{i, 3}^{*}\right)$, for $i=1,2$.

\begin{tabular}{c|c|c|c|c|c}
\hline Col. & Row & Population & $\tilde{\mathbf{z}}^{*}$ & $\widetilde{\boldsymbol{x}}_{\mathbf{1}}^{*}$ & $\widetilde{\boldsymbol{x}}_{\mathbf{2}}^{*}$ \\
\hline 21 & 31 & 1752 & $(-31295,1341,33933)$ & $(0.000,0.000,0.000)$ & $(-5.72,0.26,0.623)$ \\
21 & 32 & 1861 & $(-27081,1193,29426)$ & $(0.000,0.000,0.000)$ & $(-5.22,0.24,5.70)$ \\
21 & 33 & 2171 & $(-25452,1053,27519)$ & $(0.000,0.000,0.000)$ & $(-4.77,0.21,5.19)$ \\
21 & 34 & 2173 & $(-20265,920,22070)$ & $(0.000,0.000,0.000)$ & $(-4.32,0.21,4.73)$ \\
21 & 38 & 0 & $(0.000,0.000,0.000)$ & $(0.000,0.000,0.000)$ & $(0.00,0.00,0.00)$ \\
\hline
\end{tabular}

The fuzzy results in Table 2 have a large spread between the upper and lower limits relative to the magnitude of the central estimate. This large variation is attributable to the high degree of uncertainty attached to the model parameters and is therefore an artefact of the present hypothetical model application. Future applications of the developed fuzzy model using less variable fuzzy input parameters expected to produce more precise optimal solutions (Schultz, 2016).

The developed fuzzy model also forecasts the expected health impacts associated with the optimal solution, with Figure 3 showing the expected health impact for the present case study. Here, the expected health impacts are greatest in the mid-north and less pronounced to the south and west. This is likely attributable to the population size of these areas as health impacts are calculated based on population density (4). Overall, the health impact is low however this is a result of the two sample control strategies considered here.

Thus, the developed optimisation model has successfully identified optimal combination of control strategies within the limited context of the sample application, providing numeric results which represent the uncertainty inherent to emission analyses to an extent.

\section{CONCLUSIONS}

In this paper, a theoretical fuzzy optimisation model has been formulated, with applications to an air pollution context. The objective function of the model is to maximise the estimated, uncertain health benefits to society while accommodating for uncertainty in model parameters, to some extent, using fuzzy logic. This is a distinguishing feature of the developed model as many currently available models for air quality management focus on implementation costs only, with limited consideration of uncertainty. However, health effects are an important consideration as low-cost control strategies may not necessarily provide the greatest health benefits, leading to an increase in long-term health expenses. The consideration of uncertainty is also imperative to ensure conclusions formed on the basis of the model are well-informed.

The use of the developed fuzzy optimisation model has been demonstrated through a sample application in Adelaide, South Australia. The results indicate that the fuzzy model is able to identify a cost-effective solution within the limited context of the sample application. Results are consistent between the developed fuzzy model and the corresponding crisp model, however the fuzzy model has the additional advantageous feature of representing parameter uncertainty to some extent through the fuzzy representation of model results.

It should be noted that there are a number of complex processes impacting air pollution emissions and exposure that are beyond the scope of the developed model. The fuzzy optimisation model should therefore be viewed as a theoretical model that is a step towards the establishment of an accountable, integrated air quality management framework through the accommodation for uncertainty in model parameters. 


\section{ACKNOWLEDGMENTS}

This research would not have been possible without the continual support of the South Australian Environment Protection Authority to whom we would like to extend our sincere thanks and appreciation. The authors would also like to thank the reviewers for the invaluable comments which have enriched this research.

\section{REFERENCES}

Abt Associates Inc. (2012). Environmental Benefits Mapping and Analysis Program. Prepared for the Office of Air Quality Planning and Standards, Environmental Protection Agency of the United States of America, Research Triangle Park, NC, USA.

Australian Safety and Compensation Council (2008). The health of nations: the value of a statistical life. Australian Government Report.

Boulter, P. and K. Kulkarni (2013). Economic analysis to inform the national plan for clean air (particles). Report, Pacific Environment Ltd \& Marsden Jacob Associates. Prepared for NEPC Service Corporation.

Department of Environment and Conservation (2005). Air pollution economics: health costs of air pollution in the greater Sydney metropolitan region. Report, New South Wales Government, Sydney, Australia.

Facchinetti, G., Ricci, R., and S. Muzzioli (1998). Note on ranking fuzzy triangular numbers. International Journal of Intelligent Systems, 13, 613-622.

Gauderman, W., Avol, E., Gilliland, F., Vora, H., Thomas, D., Berhane, K., McConnell, R., Kuenzli, N., Lurmann, F., Rappaport, E., Margolis, H., Bates, D., and J. Peters (2004). The effect of air pollution on lung development from 10 to 18 years of age. The New England Journal of Medicine, 351(11), 1057-1067.

Golder Associates (2013). Exposure assessment and risk characterisation to inform recommendations for updating air quality standards for $\mathrm{PM}_{2.5}, \mathrm{PM}_{10}, \mathrm{O}_{3}, \mathrm{NO}_{2}$ and $\mathrm{SO}_{2}$. Technical Report 127643066-001-R-Rev0, submitted to National Environment Protection Council Service Corporation.

Hansen, A., Bi, P., Nitschke, M., Pisaniello, R., Sullivan, T., and A. Barnett (2012). Particulate air pollution and cardiorespiratory hospital admissions in a temperate Australian city: a case-crossover analysis. Science of the Total Environment, 416, 48-52.

Lai, Y., and C. Hwang (1992). Fuzzy Mathematical Programming. Methods and Applications. Lecture Notes in Economics and Mathematical Systems. Springer-Verlag.

Lemke, C. (1968). On complementary pivot theory. In: Dantzig, G. and Veinott, A., Mathematics of the Decision Sciences. American Mathematical Society, Providence.

Liu, L., Huang, G., Liu, Y., Fuller, G., and G. Zeng (2003). A fuzzy-stochastic robust programming model for regional air quality management under uncertainty. Engineering Optimization, 35(2), 177-199.

Lv, Y., Huang, G., Li, Y., Yang, Z., and W. Sun (2011). A two-stage inexact joint-probabilistic programming method for air quality management under uncertainty. Journal of Environmental Management, 92, 813-826.

Mesa-Frias, M., Chalabi, Z., and A. Foss (2014). Quantifying uncertainty in health impact assessment: A casestudy example of indoor housing ventilation. Environment International, 62, 95-103.

Mottaghi, A., Ezzati, R., and E. Khorram (2015). A new method for solving fuzzy linear programming problems based on the fuzzy linear complementary problem. Int. Journal Fuzzy Systems, 17, 236-245.

Oxley, T. and H. ApSimon (2011). A conceptual framework for mapping uncertainty in integrated assessment. $19^{\text {th }}$ International Congress on Modelling and Simulation, Perth, Australia, Dec. $12-16$.

Power, M., Weisskopf, M., Alexeef, S., Coull, B., Sipro, A., and J. Schwartz (2011). Traffic-related air pollution \& cognitive function in a cohort of older men. Environmental Health Perspectives, 119, $682-687$.

Qin, X., Huang, G., and L. Liu (2010). A genetic-algorithm-aided stochastic optimization model for regional air quality management under uncertainty. Journal of the Air \& Waste Management Association, 60, 63-71.

Raaschou-Nielsen, O., Sorenson, M., Ketzel, M., Hertel, O., Loft, S., Tjonneland, A., Overvad, K., and Z., Andersen (2013). Long-term exposure to traffic-related air pollution and diabetes-associated mortality: A cohort study. Diabetologia, 56, 36-46.

Schultz, L. (2016). Traffic-Related Air Pollution: Modelling Emissions and Optimal Control for Exposure Mitigation. PhD Thesis, University of South Australia, Adelaide, South Australia.

Simpson, R., Williams, G., Petroeschevsky, A., Best, T., Morgan, G., Denison, L., Hinwood, A., and G. Neville (2005). The short-term effects of air pollution on hospital admissions in four Australian cities. Australian and New Zealand Journal of Public Health, 29(3), 213-221.

Sonawane, N., Patil, R., and V. Sethi (2012). Health benefit modelling and optimization of vehicular pollution control strategies. Atmospheric Environment, 60, 193-201.

World Health Organisation (2017). Ambient (outdoor) air quality and health: fact sheet. Available online at: http://www.who.int/mediacentre/factsheets/fs313/en/. Accessed on: 12/07/2017. 